\title{
Diagnósticos de enfermagem prevalentes de pacientes em cuidados paliativos: uma mineração de dados
}

\author{
Prevalent nursing diagnosis of patients in palliative care: a data mining \\ Diagnóstico de enfermería prevalente del paciente en cuidados paliativos: una minería de datos
}

Recebido: 14/12/2021 | Revisado: 21/12/2021 | Aceito: 24/12/2021 | Publicado: 26/12/2021

\author{
Aline Tsuma Gaedke Nomura \\ ORCID: https://orcid.org/0000-0002-2584-5769 \\ Hospital das Clínicas de Porto Alegre, Brasil \\ E-mail: anomura@hcpa.edu.br \\ Miriam de Abreu Almeida \\ ORCID: https://orcid.org/0000-0002-4942-9882 \\ Hospital de Clínicas de Porto Alegre, Brasil \\ E-mail: maalmeida@hcpa.edu.br \\ Lisiane Pruinelli \\ ORCID: https://orcid.org/0000-0002-1046-6037 \\ Hospital de Clínicas de Porto Alegre, Brasil \\ E-mail: pruin001@umn.edu \\ Ana Cristina Pretto Báo \\ ORCID: https://orcid.org/0000-0002-2747-7197 \\ Hospital de Clínicas de Porto Alegre, Brasil \\ E-mail: abao@hcpa.edu.br \\ Natália Felix Gasperini \\ ORCID: https://orcid.org/0000-0003-0022-033X \\ Hospital de Clínicas de Porto Alegre, Brasil \\ E-mail: ngasperini_est@hcpa.edu.br \\ Luciana Nabinger Menna Barreto \\ ORCID: https://orcid.org/0000-0002-8166-9480 \\ Hospital de Clínicas de Porto Alegre, Brasil \\ E-mail: lnbarreto@hcpa.edu.br
}

\begin{abstract}
Resumo
Introdução: o cuidado paliativo é centrado no paciente e inclui abordagens para o alívio dos sintomas e redução fisiológica e sofrimento psíquico associado à doença. Neste contexto, o diagnóstico de enfermagem (DE) estabelece bases para a seleção de intervenções de enfermagem para alcançar resultados nesta população, pelos quais o enfermeiro é responsável. Objetivo: identificar os DE prevalentes em pacientes em cuidados paliativos, perfil sociodemográfico e clínico de pacientes adultos hospitalizados que receberam consultorias em cuidados paliativos em unidades clínicas e cirúrgicas registrados em prontuário eletrônico. Método: estudo observacional retrospectivo com uso secundário de dados. A população do estudo foi composta por todos os adultos internados nas unidades clínicas e cirúrgicas de um hospital universitário entre junho de 2014 e julho de 2019, totalizando aproximadamente 51.000 prontuários únicos. A amostra compreendeu os pacientes que receberam consultorias em cuidados paliativos durante a internação. A análise dos dados foi realizada por meio do Structured Query Language (SQL). Resultados: foram eleitos 91 diferentes diagnósticos de enfermagem distintos para a amostra do estudo. Destes, três DE foram prevalentes: Risco de quedas esteve presente na prescrição de 1350 pacientes, Integridade tissular prejudicada em 1073 prescrições e Dor aguda em 1032. Conclusão: espera-se que a metodologia adotada nesta pesquisa apoie o processo de tomada de decisão dos profissionais de saúde, a fim de melhorar a eficácia no cuidado paliativo e otimizar o processo de segurança do paciente.
\end{abstract}

Palavras-chave: Mineração de dados; Cuidados paliativos; Diagnóstico de enfermagem.

\begin{abstract}
Introduction: palliative care is patient-centered and includes approaches to symptom relief and physiological reduction and psychological distress associated with the disease. In this context, the nursing diagnosis (ND) establishes bases for the selection of nursing interventions to achieve results in this population, for which the nurse is responsible. Objective: to identify the prevalent NDs in palliative care patients, sociodemographic and clinical profile of hospitalized adult patients who received palliative care consultations in clinical and surgical units registered in electronic medical records. Method: retrospective observational study with secondary use of data. The study population consisted of all adults admitted to the clinical and surgical units of a university hospital between June 2014
\end{abstract}


and July 2019, totaling approximately 51,000 unique records. The sample comprised patients who received consultations in palliative care during hospitalization. Data analysis was performed using Structured Query Language (SQL). Results: 91 different nursing diagnoses were chosen for the study sample. Of these, three ND were prevalent: Risk of falls was present in the prescription of 1350 patients, Impaired tissue integrity in 1073 prescriptions and Acute pain in 1032. Conclusion: it is expected that the methodology adopted in this research supports the decision-making process of professionals in order to improve effectiveness in palliative care and optimize the patient safety process.

Keywords: Data mining; Palliative care; Nursing diagnosis.

\begin{abstract}
Resumen
Introducción: los cuidados paliativos se centran en el paciente e incluyen enfoques para el alivio de los síntomas y la reducción fisiológica y el malestar psicológico asociados con la enfermedad. En este contexto, el diagnóstico de enfermería (DE) establece las bases para la selección de intervenciones de enfermería para lograr resultados en esta población, de la cual la enfermera es responsable. Objetivo: Identificar los DE prevalentes en pacientes en cuidados paliativos, perfil sociodemográfico y clínico de paciente adultos hospitalizados que recibieron consultas de cuidados paliativos en unidades clínicas y quirúrgicas registradas en historias clínicas electrónicas. . Método: estudio observacional retrospectivo con uso secundario de datos. La población de estudio estuvo constituida por todos los adultos ingresados en las unidades clínicas y quirúrgicas de un hospital universitario entre junio de 2014 y julio de 2019, totalizando aproximadamente 51.000 registros únicos. La muestra estuvo constituida por pacientes que recibieron consultas en cuidados paliativos durante la hospitalización. El análisis de datos se realizó mediante el lenguaje de consulta estructurado (SQL). Resultados: se eligieron 91 diagnósticos de enfermería diferentes para la muestra de estudio. De estos, tres DE fueron prevalentes: Riesgo de caídas estuvo presente en la prescripción de 1350 pacientes, Integridad tisular deteriorada en 1073 prescripciones y Dolor agudo en 1032. Conclusión: se espera que la metodología adoptada en esta investigación apoye el proceso de toma de decisiones de profesionales para mejorar la eficacia de los cuidados paliativos y optimizar el proceso de seguridad del paciente.
\end{abstract}

Palabras clave: Minería de datos; Cuidados paliativos; Diagnóstico de enfermería.

\title{
1. Introdução
}

As informações de saúde associadas aos registros eletrônicos de saúde (RES) compreendem uma variedade de dados de saúde, que, se manuseados de maneira adequada para uma finalidade específica, fornecem orientação, instrução e conhecimento aos profissionais de saúde, sobretudo no cuidado ao paciente paliativo (Miranda et al., 2019; Plantier et al., 2017; Silva, 2015).

O cuidado paliativo é centrado no paciente e inclui abordagens para o alívio dos sintomas e redução fisiológica e sofrimento psíquico associado à doença. Esse processo concentra-se na prestação de cuidados, desenvolvendo planos que são consistentes com os objetivos dos pacientes e cuidadores, destacando as consultorias direcionadas a esta população como estratégias de cuidado (Rubens et al., 2019). Neste contexto, o diagnóstico de enfermagem (DE) estabelece base para a seleção de intervenções de enfermagem para alcançar resultados nesta população, pelos quais o enfermeiro é responsável (RabeloSilva et al., 2016). Somado a isso, as técnicas de análise de Big Data, podem contribuir com a compreensão do cumprimento das diretrizes de acordo com protocolos baseados em evidências e a influência destes sobre os resultados de pacientes em uma abordagem paliativa (Pruinelli et al., 2016).

A expressão Big Data emergiu na última década para abranger o fenômeno de grandes quantidades de dados que resultam em técnicas de pesquisas inovadoras e tecnologias de informação. Big Data é uma métrica de tamanho que reflete um conjunto de dados muito além daqueles comumente encontrados em investigação e que, atualmente, encontram-se disponíveis exigindo novas formas de gerir e novos métodos de análise, a fim de que se obtenha importância para prática (Brennan \& Bakken, 2015). Dentre as técnicas, destaca-se a mineração de dados que compreende a análise de grandes conjuntos de dados a fim de encontrar relações inesperadas e de resumir os dados de forma que eles sejam tanto úteis quanto compreensíveis para o pesquisador (Brennan \& Bakken, 2015). Metodologias baseadas em dados têm o potencial de identificar novas percepções de registros eletrônicos de saúde, direcionando rápida tomada de decisão (Pruinelli et al., 2016). 
Considerando-se o desafio do tratamento em cuidados paliativos e a necessidade de a enfermagem apropriar-se de um sistema de saúde habilitado pela da ciência de dados, buscou-se com este estudo estabelecer os DE prevalentes para pacientes internados nesta condição, fazendo uso de metodologias apropriadas para auxiliar o processo. Assim, a busca por novas alternativas visa propiciar a qualificação da avaliação multidimensional intrínseca a estes pacientes, o que ainda é uma questão em estudo.

Portanto, o objetivo deste estudo foi identificar os DE prevalentes em pacientes em cuidados paliativos, além do perfil sociodemográfico e clínico de pacientes adultos hospitalizados que receberam consultorias em cuidados paliativos em unidades clínicas e cirúrgicas registrados em prontuário eletrônico.

\section{Metodologia}

Estudo observacional retrospectivo com uso secundário de dados, que faz uso da análise de dados para direcionar o planejamento e, principalmente, o processo de tomada de decisões (Westra et al., 2016, 2018).

O estudo foi realizado a partir de dados eletrônicos oriundos de um hospital universitário do sul do país. A instituição foi uma das pioneiras no país na criação dos primeiros serviços de Tratamento de Dor e Cuidados Paliativos, sendo, hoje, referência nestas áreas. Assim, conta com uma política e plano de gerenciamento da dor e cuidados paliativos, estabelecendo estratégias educacionais para os pacientes em diferentes situações clínicas e para prevenção, recuperação e/ou tratamento dos quadros álgicos agudos ou crônicos. Para tanto, a instituição oferece protocolos para gerenciamento da dor, bem como prevê a educação aos profissionais da saúde sobre o seu manejo e gerenciamento, por estratégias presenciais e à distância anualmente. Serviços, programas e consultorias para a melhoria do cuidado ao paciente paliativo têm sido oferecidos, entre eles o Programa de Cuidados Paliativos e Enfermagem em paliativos (Sistema AGHUse - Portal Hospital de Clínicas de Porto Alegre, 2020). Cabe salientar que o hospital é acreditado pela JCI desde 2013, ou seja, diversas estratégias vêm sendo desempenhadas com o objetivo de obter e manter o selo de qualidade conquistado (Nomura et al., 2018; Nomura et al., 2016).

A população do estudo foi composta por RES de todos os adultos internados nas unidades clínicas e cirúrgicas do hospital. A extração de dados abrangeu o período entre junho de 2014 e julho de 2019, totalizando aproximadamente 51.000 prontuários únicos. Os dados dos pacientes foram identificados e liberados pela instituição. A amostra compreendeu os pacientes que receberam consultorias em cuidados paliativos durante a internação. Critérios de exclusão foram: pacientes menores de 18 anos.

Os dados do RES consistiam no conjunto de dados de tabelas interconectadas e iterativas, originando documentos como dados sociodemográficos, diagnósticos médicos e de enfermagem e de consultorias solicitadas durante a internação. A exploração dos dados foi realizada por meio de queries construídas especificamente para este estudo, a fim de extrair os dados necessários para definir e conduzir análises posteriores. Esses dados foram inseridos pelo pesquisador em ambiente Structured Query Language (SQL), a fim de automatizar, organizar e padronizar melhor os elementos dos dados e como eles se relacionam entre si e com as diferentes propriedades, gerando um banco de dados abrangente com dados brutos para posterior gerenciamento e análise. O projeto foi aprovado pelo Comitê de Ética em Pesquisa sob nº 2018.0669.

\section{Resultados}

Entre as 146 especialidades consultoras, encontram-se Enfermagem em paliativos e Programa de cuidados paliativos. A amostra foi composta por 1.727 pacientes que tiveram acompanhamento de uma destas especialidades durante sua internação, sendo que destes, 315 seguiram as consultorias em outras internações. A idade média foi de 67 anos ( $\mathrm{DP}=14,23 \mathrm{e}$ mediana $=64)$, a variação das idades permeou os extremos de 19 a 96 anos. Destes, $938(54,37 \%)$ eram do sexo masculino e 
788 (45,63\%) eram do sexo feminino. Quanto à raça, os pacientes foram classificados em 1 indígena, 3 asiáticos, $80(4,63 \%)$ pardos, $150(8,68 \%)$ negros e $1492(86,39 \%)$ brancos. Quanto ao grau de escolaridade, esses pacientes foram categorizados em: $64(3,70 \%)$ sem escolaridade alguma, 818 (47,36\%) com ensino fundamental incompleto, 290 (16,79\%) com ensino fundamental completo, 286 (16,56\%) com ensino médio incompleto, 71 (4,11\%) com ensino médio completo, 37 (2,14\%) com ensino superior incompleto, 58 (3,35\%) com ensino superior completo e $102(5,90 \%)$ sem cadastro.

Considerando os motivos de internação elencados, as prevalentes foram as neoplasias 47,79\%, doenças do aparelho respiratório 9,86\% e doenças do aparelho circulatório 9,44\% dos pacientes internados.

Foram eleitos 91 diferentes diagnósticos de enfermagem distintos para a amostra do estudo. Destes, três DE foram prevalentes: Risco de quedas esteve presente na prescrição de 1350 pacientes, Integridade tissular prejudicada em 1073 prescrições e Dor aguda em 1032.

\section{Discussão}

A avaliação acurada, completa e sistemática dos sintomas na assistência de enfermagem do paciente em cuidados paliativos é multidimensional. Neste sentido, o uso de Sistemas de Linguagem Padronizados (SLP) se apresenta como alternativa factível para qualificar a assistência de enfermagem a estes pacientes (Mello et al., 2019).

Em estudo que abordou o tema sobre taxonomias e cuidados paliativos, diferentemente do presente estudo, o sexo feminino mostrou-se predominante com 58,90\% da amostra (Xavier et al., 2019). Quanto ao grau de escolaridade, no mesmo estudo abordado, o ensino fundamental incompleto apresentou frequência de $45,21 \%$, sendo predominante, convergindo com os achados desta pesquisa (Mello et al., 2019; Xavier et al., 2019). Entende-se que o conhecimento sobre características sociodemográficas e clínicas dos pacientes e a influência que estas remetem no impacto do processo de cuidados constitui importante estratégia para qualificar o atendimento prestado, em busca da segurança do paciente.

Dessa forma, considerando os motivos de internação nesta população, as neoplasias foram prevalentes com 47,79\% da amostra. A maioria dos estudos que abordam cuidados paliativos trazem como foco de estudo pacientes oncológico (Mello et al., 2019; Xavier et al., 2019), por conviverem com um dos problemas de saúde pública mais complexos da atualidade, em que muitos casos não há possibilidades terapêuticas de cura.

A dor aguda de pacientes com câncer em cuidados paliativos pode ser constante ou intermitente causada por diversos mecanismos, como a invasão direta do tumor (local e sistêmica), resposta aos exames diagnósticos invasivos (biópsia) e às diversas terapias (cirurgia, quimioterapia ou radioterapia) (Mello et al., 2019; Sampaio et al., 2021). Um estudo explorou o diagnóstico de enfermagem dor aguda em pacientes em cuidados paliativos. O DE abrange avaliação do nível de dor nos seguintes indicadores: dor relatada, duração dos episódios de dor, expressões faciais de dor, agitação e irritabilidade (Mello et al., 2019). Entretanto, esta avaliação extrapola o nível de dor como fator indicativo de dor, sendo evidenciadas outras metas de avaliação como sinais vitais, controle da dor, sono, estado de conforto e bem-estar pessoal (Mello et al., 2019).

As quedas em pacientes hospitalizados têm associação com consequências negativas, sendo um importante problema relacionado à segurança do paciente (Marco Antonio de Goes Victor et al., 2017; Marinho et al., 2017), sobretudo quando trata-se da vulnerabilidade de um paciente em cuidados paliativos. Dessa forma, O DE risco de quedas foi identificado em uma revisão integrativa sobre taxonomias padronizadas de enfermagem e sua relação com o conceito de cuidados paliativos (Silva et al., 2020). Assim como no presente estudo, este DE foi evidenciado como importante aliado para direcionar intervenções mais acuradas, otimizando e fornecendo subsídios para formulação de planos terapêuticos para sua prevenção.

O diagnóstico de Integridade Tissular Prejudicada tem como definição: dano às membranas mucosas, córnea, pele ou tecidos subcutâneos (NANDA INTERNATIONAL NURSING DIAGNOSES: definitions \& classification., 2021), podendo ser 
evidenciado por equimoses, tecido lesado, hematoma, hiperemia, necrose tecidual. Além disso, podem apresentar diversos fatores causais como imobilização no leito, diminuição de atividade motora, falta de adequada perfusão sanguínea tissular (Silva et al., 2020). Com a evolução da doença, é comum que todas essas características se agravarem, tornando ainda mais imprescindível um olhar atento para a avaliação desse domínio que abrange a segurança e o conforto do paciente.

Em uma revisão integrativa que apontou 28 estudos sobre gerenciamento de dor em registros eletrônicos em saúde com potencial impacto clínico, pelo menos oito deles explicitaram as técnicas de extração de dados como algoritmos, processamento de linguagem natural, mineração de texto, aprendizado de máquinas e SQL (Structure Query Language) (Nomura et al., 2021). O mesmo artigo reforça que o conhecimento dos enfermeiros sobre a prática de gerenciamento clínico da dor é essencial para otimizar o RES e direcionar de forma eficaz a extração desse tipo de dados (Nomura et al., 2021).

Assim, a enfermagem tem muito a contribuir e a ganhar com um sistema de saúde habilitado pela ciência de dados e com uso de tecnologias (Gaedke Nomura et al., 2021). A prática, pesquisa e ensino em enfermagem aliados à ciência de dados, mostram-se visionários na atual conjuntura interdisciplinar e tecnológica (Gaedke Nomura et al., 2021). A contribuição da enfermagem permeia a introdução de dados de enfermagem, interpretação e análise de resultados, além de gerenciar o uso eficiente desses dados (Brennan \& Bakken, 2015).

O estudo apresentou limitações a serem consideradas. Embora a população de pacientes que estiveram internados em cuidados paliativos seja certamente maior que a apresentada no estudo, a mineração de dados limitou-se àquelas que tiveram consultorias em cuidados paliativos durante sua internação.

\section{Conclusão}

Este estudo permitiu estabelecer os três DE mais prevalentes em pacientes adultos internados em unidades clínicas e cirúrgicas de um hospital universitário do sul do país, que receberam consultorias em cuidados paliativos. Além disso, foi possível traçar o perfil sociodemográfico e clínico dessa população específica.

O conhecimento sobre o perfil do paciente em cuidados paliativos e seus diagnósticos de enfermagem prevalentes pode contribuir com diretrizes institucionais na busca de uma melhor avaliação e reavaliação, tratamento e envolvimento desse paciente vulnerável no plano de cuidados. A pesquisa fornece subsídios para a prática clínica, com um panorama dos diagnósticos de enfermagem, a fim de melhorar o cuidado e a segurança dos pacientes em cuidados paliativos.

Espera-se que a metodologia adotada nesta pesquisa apoie o processo de tomada de decisão dos profissionais de saúde, a fim de melhorar a eficácia no cuidado paliativo e, assim, otimizar o processo de segurança do paciente. Dessa forma, os resultados deste estudo permitiram o processo de extração de conhecimento derivado de dados de RES apoiando as práticas de saúde, ensino e pesquisa.

\section{Agradecimentos}

Este estudo foi financiado em parte pela Coordenação de Aperfeiçoamento de Pessoal de Nível Superior (CAPES; Agência Federal de Apoio e Avaliação da Pós-Graduação; Código Financeiro 001) e pelo Conselho Nacional de Desenvolvimento Científico e Tecnológico (CNPq; 426779 / 2018- 5). Este estudo também foi financiado pelo Fundo de Incentivo à Pesquisa e Eventos do Hospital de Clínicas de Porto Alegre (FIPE / HCPA).

\section{Referências}

Brennan, P. F., \& Bakken, S. (2015). Nursing Needs Big Data and Big Data Needs Nursing. Journal of Nursing Scholarship: An Official Publication of Sigma Theta Tau International Honor Society of Nursing, 47(5), 477-484. https://doi.org/10.1111/jnu.12159 
Research, Society and Development, v. 10, n. 17, e217101724725, 2021 (CC BY 4.0) | ISSN 2525-3409 | DOI: http://dx.doi.org/10.33448/rsd-v10i17.24725

Gaedke Nomura, A. T., de Abreu Almeida, M., Johnson, S., \& Pruinelli, L. (2021). Pain Information Model and Its Potential for Predictive Analytics: Applicability of a Big Data Science Framework. Journal of Nursing Scholarship: An Official Publication of Sigma Theta Tau International Honor Society of Nursing, 53(3), 315-322. https://doi.org/10.1111/jnu.12648

Sistema AGHUse - Portal Hospital de Clínicas de Porto Alegre. (2020.). Www.hcpa.edu.br. Retrieved December 14, 2021, from https://www.hcpa.edu.br/institucional/tecnologia-da-informacao-e-comunicacao/institucional-sistema-aghuse

NANDA INTERNATIONAL NURSING DIAGNOSES: definitions \& classification. (2021). Thieme Medical Publishers.

Marco Antonio de Goes Victor, Melissa de Freitas Luzia, Isis Marques Severo, Miriam de Abreu Almeida, Marta Georgina Oliveira de Goes, \& Amália de Fátima Lucena. (2017). Falls in Surgical Patients: Subsidies for Safe Nursing Care. Journal of Nursing UFPE / Revista de Enfermagem UFPE, 11(10), 40274035. https://doi.org/10.5205/reuol.10712-95194-3-SM.1110sup201704

Marinho, G. S., Alves, G. A. de A., Oliveira, D. F. de, Góes, Â. C. F., \& Martinez, B. P. (2017). Risco de quedas em pacientes hospitalizados. Revista Pesquisa em Fisioterapia, 7(1), 55-60. https://doi.org/10.17267/2238-2704rpf.v7i1.1218

Mello, B. S., Almeida, M. de A., Pruinelli, L., \& Lucena, A. de F. (2019). Resultados de enfermagem para avaliação da dor de pacientes em cuidado paliativo. Revista Brasileira de Enfermagem, 72, 64-72. https://doi.org/10.1590/0034-7167-2018-0307

Miranda, G. M., Rosa, L. M. da, Bertoncello, K. C. G., Mercês, N. N. A. das, Amante, L. N., \& Alvarez, A. G. (2019). Sistema informatizado à decisão clínica em Enfermagem: Uma construção e validação na oncologia. Enferm. foco (Brasília), 103-108.

Nomura, A. T. G., Pruinelli, L., Barreto, L. N. M., Graeff, M. dos S., Swanson, E. A., Silveira, T., \& Almeida, M. de A. (2021). Pain Management in Clinical Practice Research Using Electronic Health Records. Pain Management Nursing, 22(4), 446-454. https://doi.org/10.1016/j.pmn.2021.01.016

Nomura, A. T. G., Pruinelli, L., da Silva, M. B., Lucena, A. de F., \& Almeida, M. de A. (2018). Quality of Electronic Nursing Records: The Impact of Educational Interventions During a Hospital Accreditation Process. Computers, Informatics, Nursing: CIN, 36(3), $127-132$. https://doi.org/10.1097/CIN.0000000000000390

Nomura, A. T. G., Silva, M. B. da, \& Almeida, M. de A. (2016). Quality of nursing documentation before and after the Hospital Accreditation in a university hospital. Revista Latino-Americana de Enfermagem, 24. https://doi.org/10.1590/1518-8345.0686.2813

Plantier, M., Havet, N., Durand, T., Nicolas, C., Amaz, C., Biron, P., Philip, I., \& Perrier, L. (2017). Does adoption of electronic health records improve the quality of care management in France? Results from the French e-SI (PREPS-SIPS) study. International Journal of Medical Informatics, 102, 156-165. https://doi.org/10.1016/j.ijmedinf.2017.04.002

Pruinelli, L., Yadav, P., Hangsleben, A., Johnson, J., Dey, S., McCarty, M., Kumar, V., Delaney, C. W., Steinbach, M., Westra, B. L., \& Simon, G. J. (2016). A Data Mining Approach to Determine Sepsis Guideline Impact on Inpatient Mortality and Complications. AMIA Summits on Translational Science Proceedings, 2016, 194-202.

Rabelo-Silva, E., Cavalcanti, A., Goulart, M., Lucena, A., Almeida, M., Linch, G., Silva, M., \& Müller-Staub, M. (2016). Advanced Nursing Process Quality: Comparing International Classification for Nursing Practice (ICNP) with the NANDA-International (NANDA-I) and Nursing Interventions Classification (NIC). Journal of Clinical Nursing, 26. https://doi.org/10.1111/jocn. 13387

Rubens, M., Ramamoorthy, V., Saxena, A., Das, S., Appunni, S., Rana, S., Puebla, B., Suarez, D. T., Khawand-Azoulai, M., Medina, S., \& Viamonte-Ros, A. (2019). Palliative Care Consultation Trends Among Hospitalized Patients With Advanced Cancer in the United States, 2005 to 2014 . The American Journal of Hospice \& Palliative Care, 36(4), 294-301. https://doi.org/10.1177/1049909118809975

Sampaio, S., Motta, L., \& Caldas, C. (2021). Dor e Internação Hospitalar em Cuidados Paliativos. Revista Brasileira de Cancerologia, 67, e-131180. https://doi.org/10.32635/2176-9745.RBC.2021v67n3.1180

Silva, D. E. S. da, Pacheco, P. Q. C., \& Souza, S. R. de. (2020). Palliative care and its relationship with the nursing diagnoses of NANDA-I and NIC taxonomies / Cuidados paliativos e sua relação com os diagnósticos de enfermagem das taxonomias NANDA-I e NIC. Revista de Pesquisa Cuidado é Fundamental Online, 12, 282-291. https://doi.org/10.9789/2175-5361.rpcfo.v12.8548

Silva, J. dos S. (2015). Audit in health: A new paradigm in the quality of nursing care. Rev Enferm UFPI, 4(2), Article 2. https://periodicos.ufpi.br/index.php/reufpi/article/view/273

Westra, B. L., Christie, B., Johnson, S. G., Pruinelli, L., LaFlamme, A., Park, J. I., Sherman, S. G., Byrne, M. D., Ranallo, P., \& Speedie, S. (2016). Expanding Interprofessional EHR Data in i2b2. AMIA Joint Summits on Translational Science Proceedings. AMIA Joint Summits on Translational Science, 2016, 260268.

Westra, B. L., Johnson, S. G., Ali, S., Bavuso, K. M., Cruz, C. A., Collins, S., Furukawa, M., Hook, M. L., LaFlamme, A., Lytle, K., Pruinelli, L., Rajchel, T., Settergren, T. T., Westman, K. F., \& Whittenburg, L. (2018). Validation and Refinement of a Pain Information Model from EHR Flowsheet Data. Applied Clinical Informatics, 9(1), 185-198. https://doi.org/10.1055/s-0038-1636508

Xavier, É. de C. L., Júnior, A. J. S. C., Carvalho, M. M. C. de, Lima, F. R., \& Santana, M. E. de. (2019). Diagnósticos de enfermagem em cuidados paliativos oncológicos segundo diagrama de abordagem multidimensional. Enfermagem Em Foco, 10(3). https://doi.org/10.21675/2357-707X.2019.v10.n3.2109 